\title{
Effect of Liposomal Encapsulation on the Chemical Exchange Properties of Diamagnetic CEST Agents
}

\author{
Eleni Demetriou, ${ }^{*},, \S$ Harriet E. Story, ${ }^{\ddagger, \S}$ Robin Bofinger, ${ }^{\ddagger}$ Helen C. Hailes, ${ }^{\ddagger}$ Alethea B. Tabor, ${ }^{*},+\infty$ \\ and Xavier Golay ${ }^{\dagger}$ \\ ${ }^{\dagger}$ Brain Repair \& Rehabilitation, Queen Square Institute of Neurology, University College London, London WC1N 3BG, United \\ Kingdom \\ ${ }^{\ddagger}$ Department of Chemistry, University College London, 20 Gordon Street, London WC1H 0AJ, United Kingdom
}

Supporting Information

ABSTRACT: Exogenous chemical exchange saturation transfer (CEST) contrast agents such as glucose or 2-deoxy-D-glucose (2-DG) have shown high sensitivities and significant potential for monitoring glucose uptake in tumors with MRI. Here, we show that liposome encapsulation of such agents can be exploited to enhance the CEST signal by reducing the overall apparent exchange rate. We have developed a concise analytical model to describe the liposomal contrast dependence on several parameters such as $\mathrm{pH}$, temperature, irradiation amplitude, and intraliposomal water content. This is the first study in which a model has been constructed to measure the exchange properties of diamagnetic CEST agents encapsulated inside liposomes. Experimentally measured exchange rates of glucose and 2-DG in the liposomal system were found to be reduced due to the intermembrane exchange between the intra- and extraliposomal compartments because of

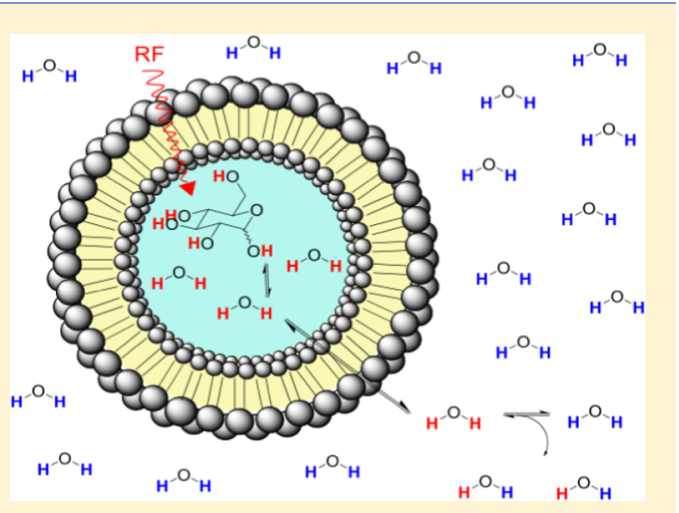
restrictions in water transfer imposed by the lipid membrane. These new theoretical and experimental findings will benefit applications of diamagnetic liposomes to image biological processes. In addition, combining this analytical model with measurements of the CEST signal enhancement using liposomes as a model membrane system is an important new general technique for studying membrane permeability.

\section{INTRODUCTION}

Over the last 10 years, many chemical exchange saturation transfer (CEST) studies have explored different approaches to enhance the sensitivity of contrast agent detection by either optimizing the exchange rate $\left(k_{\mathrm{ex}}\right)$ of the exchangeable protons in the agent with water or by increasing the number of exchangeable protons per molecule of the contrast agent. A key advantage of this technique, compared to other imaging modalities, such as position emission tomography (PET), is that it avoids the use of radiolabeled tracer molecules. In principle, small, diamagnetic molecules such as amino acids or sugars can be used for CEST. However, the detection threshold is in the millimolar range, which limits the clinical potential for these agents.

Glucose and glucose analogues are generally biocompatible and possess several exchangeable hydroxyl protons. Moreover, the increased uptake of glucose into tumors has been successfully exploited by ${ }^{18}$ F-labeled fluoro-deoxy-D-Glucose (FDG) PET imaging for over 30 years. Glucose and its analogues are, therefore, obvious candidates for development into CEST contrast agents. Nonradiolabeled D-glucose has been used at physiologically acceptable quantities to image glucose accumulation in mouse xenografts and successfully discriminate between distinct tumor phenotypes using CEST techniques (termed GlucoCEST). ${ }^{2-4}$ However, drawbacks of the technique include low sensitivity, short duration of the signal, and the potential for adverse side effects in response to a large bolus injection of glucose such as hyperglycaemic coma in diabetic patients or deep vein thrombosis. 3,4 2-Deoxy-Dglucose (2-DG) is an analogue of glucose in which the 2hydroxyl group is replaced by hydrogen, resulting in several interesting biological consequences. 2-DG enters cells via the same transporters as glucose, mainly GLUT1 and GLUT3 in the brain. ${ }^{3}$ Once inside the cell, it is phosphorylated by hexokinase to form 2-deoxyglucose-6-phosphate (2-DG6P), which is only minimally accepted as a substrate by glucose-6phosphate dehydrogenase and glucose-6-phosphate isomerase, preventing metabolism via both the oxidative pentose phosphate pathway and the glycolytic pathway, respectively. ${ }^{3}$ 2-DG6P and its metabolites have poor cell permeability and, thereby, become trapped and accumulate intracellularly, much like FDG and its metabolic products. ${ }^{5}$ The utility of 2-DG as a CEST agent has been investigated in a number of animal studies, ${ }^{3,5,6}$ and doses of up to $0.3 \mathrm{~g} / \mathrm{kg}$ have been administered in humans with limited adverse side effects.

Received: March 11, 2019

Revised: July 22, 2019 
We hypothesized that the current challenges of sensitivity and toxicity opposing the clinical translation of gluco- and 2DG-CEST could be overcome by encapsulating high concentrations of the monosaccharides in the aqueous interior of liposomes. The design of liposomal systems encapsulating paramagnetic shift reagents in the aqueous interior (termed lipoCEST) has given rise to the highest sensitivity CEST contrast agents, so far reported. In these systems, the presence of the unpaired electrons on the paramagnetic agent induces a shift of the water proton frequency inside the liposomes away from that of the surrounding water, the nature of which depends on the structure and concentration of the shift reagent. Using liposome concentrations as low as $90 \mathrm{pM}$ and by selectively irradiating the intraliposomal water pool, which acts as a reservoir containing a huge number of exchangeable protons (ranging from millions to billions of water molecules), greater MR sensitivity can be attained. ${ }^{8-10}$ Liposomes can also encapsulate vast quantities of hydrophilic molecules with exchangeable protons in the aqueous interior, allowing high local concentrations of DIACEST contrast agents to be delivered and shielding patients from potential off-target effects and toxicity of these agents. ${ }^{9,10}$

For applications in cancer imaging and cancer chemotherapy, liposomes with a diameter of $100-200 \mathrm{~nm}$ passively target tumors by passing through the fenestrations in disorganized tumor vasculature and then accumulate there due to poor lymphatic drainage (a process termed the enhanced permeability and retention (EPR) effect). ${ }^{11}$ Furthermore, peptides and antibodies with a high affinity for surface proteins and glycoproteins expressed by target cells can be covalently anchored to the liposome surface to achieve highly cell-selective delivery of contrast agents. ${ }^{12}$ Thus, selective liposomal delivery to tumors serves to reduce the toxicity of such imaging and chemotherapeutic agents to noncancerous cells and tissues, and liposomal formulations such as DOXIL (PEGylated liposomal doxorubicin) are now in clinical use. ${ }^{13}$

It is necessary, however, to understand the behavior and CEST signal characteristics of these new compounds prior to their use in vivo. The surface charge, size, and mechanical properties of the liposome itself can be fine-tuned to optimize pharmacokinetics, biodistribution, and encourage the accumulation of the contrast agent within the tissue of interest. The chemical exchange between glucose hydroxyl protons and water protons belongs to the fast-intermediate exchange regime. ${ }^{14}$ However, according to the chemical exchange theory, to generate substantial CEST contrast, the exchange rate with water must be slow enough that the slow-tointermediate exchange condition is not violated (i.e., frequency offset $\left.\Delta \omega \geq k_{\text {ex }}\right)$. $^{1}$ Because the water exchange across the membrane is very slow compared to the exchange rates in glucose, we hypothesized that the CEST effect will be quenched. ${ }^{8,9}$ Therefore, the sensitivity of GlucoCEST would be enhanced if the exchange between glucose hydroxyl protons and bulk water protons could be reduced. In turn, a more gradual release of glucose from liposomes in vivo could be used to produce a sustained CEST contrast and circumvent metabolic processes and other off-target effects.

The aim of this work was to investigate the dependency of the CEST signal generated by liposomes encapsulating high concentrations of glucose or $2-\mathrm{DG}$ on several variables such as $\mathrm{pH}$, lipid composition, temperature, and $\mathrm{RF}$ irradiation power. The differences between glucose vs. 2-DG and the free monosaccharides vs. liposome-encapsulated monosaccharides were also analyzed. A better understanding of the effects of these parameters on the resultant CEST signal enabled the construction of a full six-site exchange model for quantifying the exchange rate between monosaccharides and intraliposomal water as a function of the intraliposomal and extraliposomal water magnetization. This information can be used, in turn, to characterize the liposomal system encapsulating monosaccharides and to optimize the experimental parameters to achieve superior saturation efficiency. In addition, these liposomal systems might be employed as a novel way to estimate the exchange rate of water across lipid bilayers.

A variety of experimental and computational approaches have previously been used to study the permeability of water across membranes, including molecular dynamics, ${ }^{15}$ electrochemical sensors, monitoring changes in osmolarity, ${ }^{16}$ measurement of the self-quenching of liposomal carboxyfluorescein, ${ }^{17}$ and NMR experiments using water relaxation times to determine the exchange of water between magnetically different sites across a bilayer. ${ }^{8-10}$ The latter approach has been used with CEST spectra instead of NMR proton spectra to improve its sensitivity. ${ }^{18,19}$ In particular, the intermembrane exchange rates were calculated by fitting MTRasym spectra as a function of presaturation time. Depending on the size of the vesicles, the rate across the lipid bilayer was found to range between 13 and $221 \mathrm{~Hz}^{18}$ However, these approaches have limitations: techniques that rely on stopped-flow measurements cannot be used to study very fast rates of permeation, and lipoCEST measurements rely on the encapsulation of paramagnetic shift reagents. The liposomal systems described in this work will be useful and straightforward systems to explore the effects of different lipid compositions and biologically relevant molecules on the water permeability of membranes.

\section{THEORY}

For a system consisting of liposomes in water encapsulating monosaccharides such as glucose or 2-DG (Figure 1), the phospholipid bilayer entraps a high concentration of monosaccharide in the aqueous interior cavity. Monosaccharides do not permeate the lipid bilayer, whereas the extraliposomal water enters the intraliposomal space via the membrane and exchanges with the intraliposomal water. To be able to study the secondary effect of the water exchange across the bilayer on the primary exchange of monosaccharides with intraliposomal water, we considered two systems: a free monosaccharide solution in water and monosaccharides encapsulated inside liposomes. The exchange rate $k_{\mathrm{ex}}$ from a solute to water can be measured from a magnetization transfer experiment by selectively irradiating at the frequency offset $(\Delta \omega)$ of the glucose or 2-DG exchangeable protons with respect to the bulk water and observing the reduction of the water signal intensity as a function of saturation time or saturation power. When prolonged saturation periods are used, the saturated protons are replaced by nonsaturated water protons, which, in turn, can be saturated and exchanged providing an amplification process for the small solute signal, giving rise to detectable decreases in the water signal.

Five-Site Exchange for Pulsed RF Irradiation. In a first step, a five-site exchange model between water and glucose can be established (i.e., pool A, is the abundant water and pools B, $\mathrm{D}, \mathrm{E}, \mathrm{F}$ consist of the $\mathrm{OH}$ groups on monosaccharides 


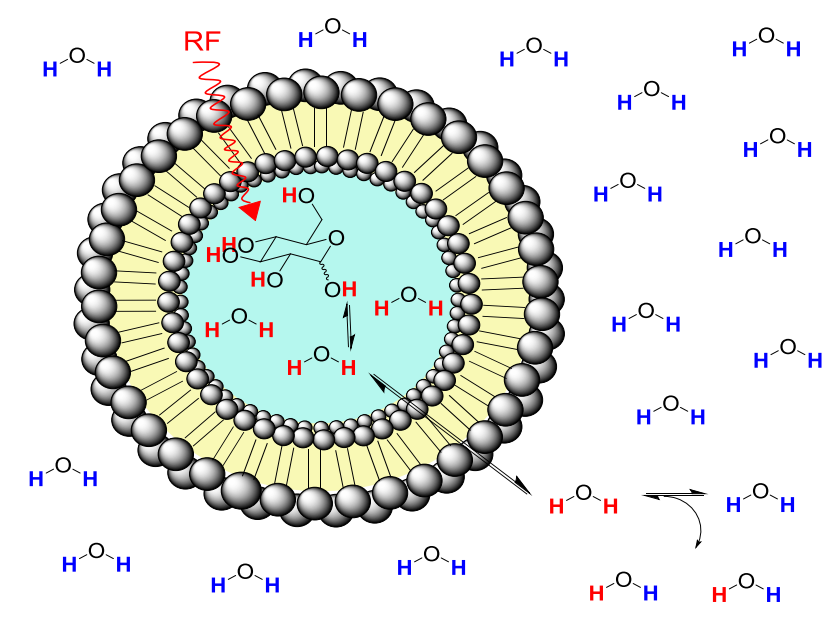

Figure 1. Schematic for diaCEST liposome encapsulating glucose. Narrow bandwidth RF pulses saturate hydroxyl protons, which undergo exchange with intraliposomal water protons. Water molecules bearing saturated protons can permeate the lipid membrane and transfer a saturated proton to a bulk water molecule. The magnitude of generated contrast depends on several variables such as liposome size, lipid composition, $\mathrm{pH}$, temperature, and irradiation power.

encapsulated inside the liposomes, which are in chemical exchange with water). Because the resonance frequency of the water protons inside (intraliposomal) and outside (bulk) the liposomes is the same and there is no significant difference between the relaxation constants (i.e., longitudinal or transverse relaxation) of the liposomal system encapsulating sugars comparative to the system of free sugars in water, both the intra- and extraliposomal water represent pool A for this system. The only difference between free and encapsulated monosaccharides is, therefore, the presence of the lipid membrane. By measuring the exchange rates separately for both systems, we can evaluate the effect of the intermembrane exchange on the total exchange rate between monosaccharides and water at different conditions.

After a prolonged period of RF saturation on resonance with glucose or 2-DG hydroxyl protons, the system reaches steady state and this simplifies the dynamics of the magnetization of this 5-pool system, which is described by the BlochMcConnell (BM) equations. ${ }^{20}$ Here, a pulsed CEST experiment will be used, as opposed to a continuous one. In such a pulsed CEST experiment, saturation pulses are separated by periods without RF irradiation, and depending on whether we assume chemical exchange during the delay or not, there are two analytical models, which can be used to describe the decay of the $z$-component of the magnetization. Under the assumption that the signal during off-resonance irradiation of duration $t_{\mathrm{p}}$ evolves at a rate $R_{1 \rho}$ (the relaxation rate along the effective field) and recovers with $R_{1 A}$ during the interpulse delay $t_{\mathrm{d}}$, the steady-state $M_{\mathrm{wz}}^{\mathrm{ss}}$ is given by ${ }^{21}$

$$
\frac{M_{\mathrm{wz}}^{\mathrm{ss}}(\Delta \omega)}{M_{0}}=\frac{R_{1 A}}{\bar{R}_{1 \rho}(\Delta \omega) \mathrm{DC}+R_{1 A}(1-\mathrm{DC})}
$$

where DC is the duty cycle defined as DC $=\frac{t_{\mathrm{p}}}{\left(t_{\mathrm{p}}+t_{\mathrm{d}}\right)}$. For shaped RF pulses, $R_{1 \rho}$ is described by the average $\overline{R_{1} \rho}$, defined previously. ${ }^{21}$

Six-Site Chemical Exchange for diaCEST Liposomes. Although a five-site model may allow an indirect effect of the lipid bilayers on the water exchange properties, the complete system can only be described through a full six-site exchange model. This model can be described as follows: the hydroxyl protons in monosaccharides are in chemical exchange with the intraliposomal water, which represents a five-site exchange (eq 1). Then, the intraliposomal water for which magnetization has been saturated through CEST effects from $-\mathrm{OH}$ protons of glucose or 2-DG enters the extraliposomal space and, thereby, transfers saturated protons to bulk water via the lipid membrane with a rate known as the intermembrane exchange rate. For the derivation of the extraliposomal magnetization, a system of six differential BM equations coupled with the intermembrane exchange rate is considered. At steady-state conditions, the differential terms become zero, and we also set the transverse magnetization terms coupled with the intermembrane exchange rate to zero without the loss of generality. Then, the solution of a system consisting of six linear equations gives the extraliposomal water magnetization as a function of the intraliposomal magnetization (see Supporting Information (SI), Section I for derivation)

$$
M_{\text {wzextralip }}^{\text {ss }}=\frac{1}{\left(R_{1}^{\text {extra }}+R_{\text {interm }} M_{0}^{\text {intra }}\right)} \cdot\left(R_{\text {interm }} M_{0}^{\text {extra }} M_{\text {wzintra }}^{\text {ss }}(\Delta \omega)+R_{1}^{\text {extra }} M_{0}^{\text {extra }}\right)
$$

where

$$
R_{\text {interm }} M_{0}^{\text {intra }}=k_{\text {extra } / \text { intra }} \text {, and } R_{\text {interm }} M_{0}^{\text {extra }}=k_{\text {intra } / \text { extra }}
$$

The measured signal from the liposomal sample is the sum of the intraliposomal and extraliposomal water magnetizations weighted by their equilibrium magnetizations $M_{0}^{\text {intra }}$ and $M_{0}^{\text {extra }}$. To calculate the intermembrane exchange rate, we fit the sum $M_{\text {wzextralip }}^{\text {ss }}+M_{\text {wzintra }}^{\text {ss }}$ at various $B_{1}$ irradiation amplitudes for both glucose or 2-DG encapsulated inside the liposomes. Finally, we compare the exchange rates of the monosaccharides calculated using a six-site model with the already established five-site model.

Calculation of Liposomal Volume. A mathematical model developed by $\mathrm{Xu}$ et al. ${ }^{22}$ can be used to calculate the total interior volume of liposome samples. The model requires the following parameters: particle diameter and size distribution (measured by dynamic light scattering (DLS)), the bilayer thickness $(d)$, the average surface area per lipid on the bilayeraqueous phase interface, and the lipid concentration $(30 \mathrm{mM}$ for all samples). The literature values used for $d$ were measured by surface force apparatus and refractive index at $21{ }^{\circ} \mathrm{C}$ and were $4.6 \mathrm{~nm}$ for 1,2-dipalmitoyl-sn-glycero-3-phosphocholine (DPPC) and $5.1 \mathrm{~nm}$ for 1,2-distearoyl-sn-glycero-3-phosphocholine (DSPC). ${ }^{23}$ The average area per lipid $(A)$ for both DPPC and DSPC bilayers was found to be $47.3 \AA^{2}$ when measured at $25{ }^{\circ} \mathrm{C}$ in the gel state, which is approximately equal to the headgroup steric limit of about $48 \AA^{2} \cdot{ }^{24,25} \mathrm{X}$-ray diffraction studies have shown that increases in chain length of disaturated phosphatidylcholines (chain lengths 16-24) have a negligible effect on $A$ which is in agreement with the head groups already being pushed to the steric repulsive limit, thus the extra van der Waals attractions contributed by additional methylene groups are not able to compress the head groups any closer together. ${ }^{24,25}$ Inputting these parameters for our liposomal samples produces calculated internal volumes in the range of $10-13 \%$. The remaining $87-90 \%$ volume is assumed to be the extraliposomal water. The interior volume for each liposome sample and the parameters used to calculate the 
Table 1. Liposome Sample Bilayer Composition, Monosaccharide Contents, Diameter, Hydrodynamic Size Distribution, Overall and Exterior Monosaccharide Concentrations and $\mathrm{pH}$

\begin{tabular}{|c|c|c|c|c|c|c|}
\hline $\begin{array}{l}\text { liposome } \\
\text { sample }\end{array}$ & $\begin{array}{l}\text { lipid bilayer } \\
(30 \mathrm{mM})\end{array}$ & $\begin{array}{l}\text { monosaccharide } \\
\text { encapsulated }\end{array}$ & $Z$-ave $(d, \mathrm{~nm})($ std dev $)$ & PdI (std dev) & $\begin{array}{c}\text { overall }[\text { monosaccharide }](\mathrm{mM}) \text { (of which } \\
\text { exterior }(\mathrm{mM}))\end{array}$ & $\mathrm{pH}$ \\
\hline 1 & DPPC & glucose & $180(0.7)$ & $0.10(0.003)$ & $24(0.3)$ & 7 \\
\hline 2 & DPPC & $2-\mathrm{DG}$ & $178(1.7)$ & $0.14(0.01)$ & $36(0.3)$ & 7 \\
\hline 3 & DPPC & glucose & $168(3.2)$ & $0.23(0.01)$ & $33(1.7)$ & 6 \\
\hline 4 & DPPC & glucose & $155(1.8)$ & $0.15(0.01)$ & $32(2.3)$ & 7 \\
\hline 5 & DPPC & $2-\mathrm{DG}$ & $151(2.6)$ & $0.18(0.01)$ & $24(0.7)$ & 6 \\
\hline 6 & DPPC & $2-\mathrm{DG}$ & 184 (1.0.) & $0.11(0.05)$ & $34(1.1)$ & 7 \\
\hline 7 & DSPC & glucose & $147(2.4)$ & $0.11(0.01)$ & $30(2.0)$ & 6 \\
\hline 8 & DSPC & glucose & $146(1.5)$ & $0.10(0.01)$ & $29(1.0)$ & 7 \\
\hline 9 & DSPC & 2-DG & $152(2.8)$ & $0.11(0.01)$ & $35(1.2)$ & 6 \\
\hline 10 & DSPC & 2-DG & $164(2.6)$ & $0.18(0.02)$ & $34(1.4)$ & 7 \\
\hline
\end{tabular}

values employing the model by $\mathrm{Xu}$ et $\mathrm{al}^{22}$ are listed in the Supporting Information (Table S1).

\section{EXPERIMENTAL SECTION}

NMR Experiments. Proton magnetic resonance spectra $\left({ }^{1} \mathrm{H} \mathrm{NMR}\right)$ were recorded at $600 \mathrm{MHz}$ on a Bruker Avance spectrometer at room temperature with suppression of water. Glucose and 2-DG samples were made up at $500 \mathrm{mM}$ in water with $20 \%$ phosphate-buffered saline (PBS) (Sigma), and the $\mathrm{pH}$ was adjusted with $1 \mathrm{M} \mathrm{HCl}$ or $\mathrm{NaOH}$ solution.

Preparation of Monosaccharide Encapsulating Liposomes. 1,2-Dipalmitoyl-sn-glycero-3-phosphocholine (DPPC) and 1,2-distearoyl-sn-glycero-3-phosphocholine (DSPC) were purchased from Anatrace, Inc. (Maumee, Ohio) and Avanti Polar Lipids (Alabaster, AL), respectively. Liposomes were formed via the thin-film hydration method with sonication. Lipid was dissolved in $\mathrm{CHCl}_{3}$, and the solvent was removed under reduced pressure to form a thin film. Thin films were hydrated with solutions of $0.5 \mathrm{M}$ glucose or $0.5 \mathrm{M}$ 2-DG in deionized (DI) water with varying percentages of freshly prepared PBS buffer at the reported $\mathrm{pH}$ and subsequently sonicated. Sonication was carried out for 10 $\min$ at a temperature higher than the relevant lipid melting transition temperature (using a VWR ultrasonic cleaner) to give polydisperse liposomes. The overall lipid concentration in liposome samples was made up to be $30 \mathrm{mM}$, and the $\mathrm{pH}$ was controlled via the addition of $1 \mathrm{M} \mathrm{HCl}$ or $\mathrm{NaOH}$ solution. The liposomes were sized by extrusion, which was carried out using a LIPEX $10 \mathrm{~mL}$ extruder with a thermobarrel (Northern Lipids Inc.) to create unilamellar vesicles. The liposomes were passed through polycarbonate membranes with a pore size of $0.4 \mu \mathrm{M}$ followed by $0.2 \mu \mathrm{M}$ to give liposome samples with low polydispersity (approaching 0.1) and diameters in the range 146-184 nm. Minimal lipid loss was observed during extrusion, therefore lipid concentrations were assumed to remain constant. Liposomes were dialyzed into $0.25 \mathrm{M} \mathrm{NaCl}$ solution with various PBS concentrations at the relevant $\mathrm{pH}$. Dialysis solutions were changed 3 times over a $32 \mathrm{~h}$ period.

Characterization of Liposome Samples. Size. The hydrodynamic size distribution of each liposome sample, following dialysis into $\mathrm{PBD} /$ water, was measured via dynamic light scattering (DLS) with a $633 \mathrm{~nm} \mathrm{He-Ne} \mathrm{laser} \mathrm{light}$ source, (Malvern Ltd. Zetasizer Nano Series ZS, Worchester, U.K.) according to the recommendations of the manufacturer. Samples were prepared by diluting $5 \mu \mathrm{L}$ of aliquots of the liposome sample in $1 \mathrm{~mL}$ of DI water that was previously passed through a $0.45 \mu \mathrm{M}$ poly(vinylidene difluoride) syringe filter. DLS measurements were taken in triplicate at $25{ }^{\circ} \mathrm{C}$ using clear $1 \mathrm{~mL} \zeta$-potential cuvettes. The mean diameter and polydispersity index (PdI) are reported for liposomal samples in Table 1.

Determining Monosaccharide Concentrations of Liposomal Samples. Overall concentrations, exterior glucose and 2-DG concentrations for liposome formulations were obtained using the Glucose GO Assay Kit supplied by SigmaAldrich. This is an enzymatic, colorimetric assay intended to measure glucose concentration, however glucose oxidase is reported to catalyze the oxidation of $2-\mathrm{DG}$ at a rate which is approximately $12 \%$ of that for glucose. ${ }^{26}$ Linear calibration curves for glucose or 2-DG were constructed in combination with each assay performed. Test and calibration solutions were made up to a total volume of $0.5 \mathrm{~mL}$. Calibration solutions were made using $0-40 \mu \mathrm{L}$ of a $1 \mathrm{mg} / \mathrm{mL}$ solution of monosaccharide diluted to a total volume of $0.5 \mathrm{~mL}$ in DI water. Test solutions for overall monosaccharide concentration for liposomal samples consisted of the liposomal sample (5 $\mu \mathrm{L}), 3 \%$ Triton X-100 (5 $\mu \mathrm{L})$, and DI water $(490 \mu \mathrm{L})$. The assay reagent conditions were found to cause monosaccharide leakage from liposomes; therefore, to measure exterior monosaccharide concentrations of liposome samples, a small aliquot of sample was centrifuged at $4000 \mathrm{rpm}$ for $5 \mathrm{~min}$. The supernatant $(5 \mu \mathrm{L})$ was then carefully removed and diluted in DI water $(495 \mu \mathrm{L})$ to create the exterior concentration test solution. The assay reagent $(1.0 \mathrm{~mL})$ was added to each calibration or test solution and agitated for $30 \mathrm{~min}$ at room temperature via shaking on an IKA KS130 basic platform shaker at $320 \mathrm{rpm}$. After this time, $6 \mathrm{M} \mathrm{H}_{2} \mathrm{SO}_{4}(1.0 \mathrm{~mL})$ was added to terminate the reaction. The absorbance at $540 \mathrm{~nm}$ was measured for the test, and calibration solutions and unknown concentration values were derived using the calibration curves. More details on how the Glucose GO Assay Kit was adapted to measure overall and exterior glucose and 2-DG concentrations of liposome preparations is provided in the Supporting Information, Section III.

MRI Protocol. All samples were imaged on a 9.4 T Agilent MRI scanner using a transmit/receive RF coil with $33 \mathrm{~mm}$ inner diameter (Rapid Biomedical, Germany). CEST measurements were acquired using a single-shot spin-echo (SE) echo planar imaging $(\mathrm{TR}=65.3 \mathrm{~ms}, \mathrm{TE}=4.07 \mathrm{~ms}, \mathrm{FOV}=20 \times 20$ $\mathrm{mm}^{2}$, slice thickness $=5 \mathrm{~mm}$, matrix size $\left.=64 \times 64\right)$ with a saturation train prior to the readout consisting of 151 Gaussian pulses at 11 irradiation amplitudes: 16.61, 32.36, 49.82, 66.85, 83.46, 100.06, 116.66, 133.27, 149.87, $183.51 \mathrm{~Hz}$, (pulse length $=50 \mathrm{~ms}, \mathrm{FA}=300,600,900,1200,1500,1800,2100$, 


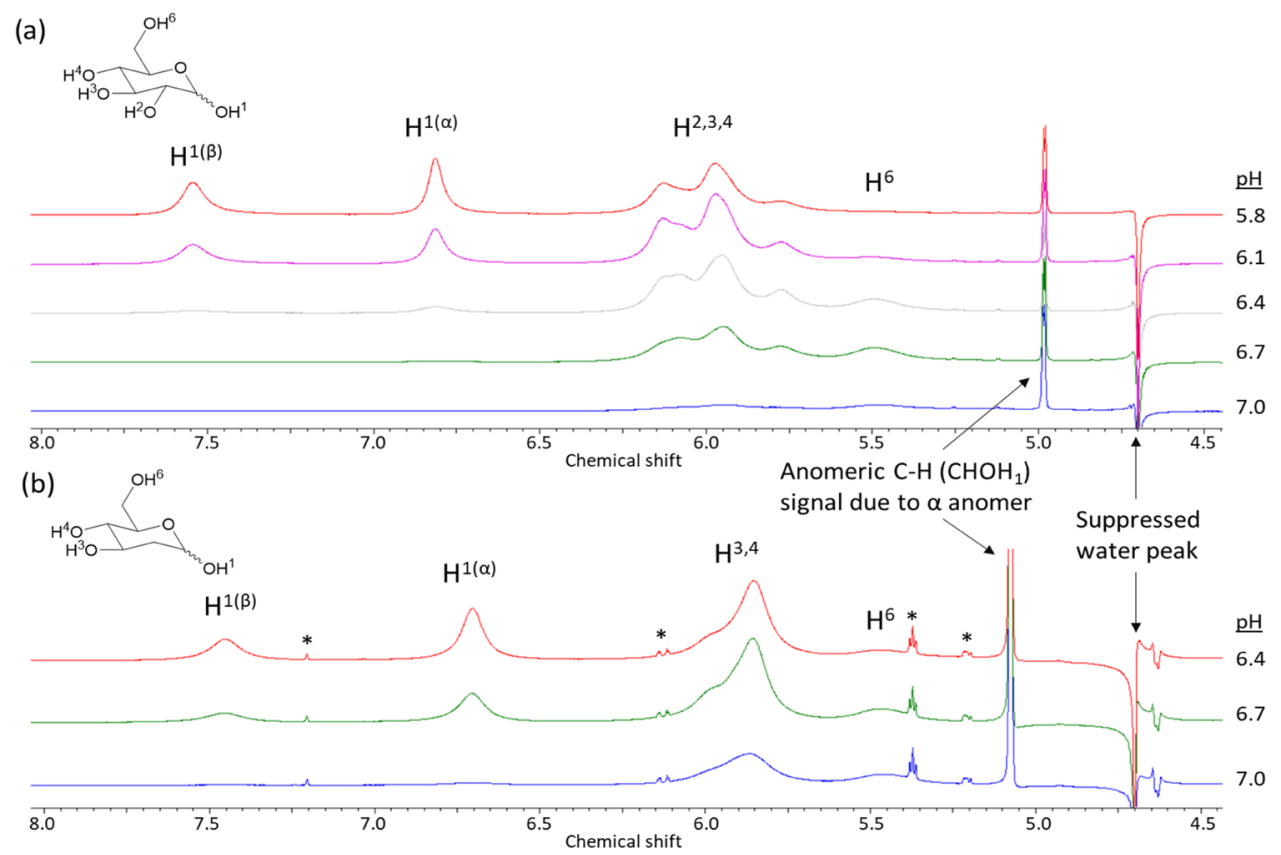

Figure 2. ${ }^{1} \mathrm{H}$ NMR spectra of $0.5 \mathrm{M}$ monosaccharides in DI water with $20 \% \mathrm{PBS}$ at various $\mathrm{pH}$ values, (a) $0.5 \mathrm{M}$ glucose at $\mathrm{pH}$ values 5.8, 6.1, 6.4, 6.7, and 7.0, (b) 0.5 M 2-DG at $\mathrm{pH}$ values 6.4, 6.7, and 7.0. The suppressed water and anomeric $\mathrm{C}-\mathrm{H}$ signals are labeled, and asterisks mark impurities present in the commercially available 2-DG.

$2400,2700,3000^{\circ}, 90 \%$ duty cycle). The sequence was repeated for 77 frequency offsets ranging between $\pm 6.08 \mathrm{ppm}$ with steps of $0.16 \mathrm{ppm}(64 \mathrm{~Hz})$. Frequencies were altered from positive to negative to compensate for potential $B_{0}$ field drifts during the acquisition.

MTRasym and Exchange Rate Measurements. Analytical solutions of the BM equations describing a five-site exchange system (eq 1) or a six-site exchange system (eqs 2 and 3) were fitted in MATLAB to calculate the exchange rates using the optimization function lsqcurvefit. The peak assignment of the hydroxyl protons in glucose or 2-DG was done by means of NMR and the frequency offsets, and relative proton concentrations were provided as fixed parameters to the fit. In addition, the longitudinal relaxation rate of water in the presence of monosaccharides was measured from a separate acquisition, and it was used for fitting. For the five-site exchange model, the normalized water magnetization at 77 frequency offsets ranging between $\pm 6.08 \mathrm{ppm}$ was fitted at 11 irradiation amplitudes (see MRI Protocol) using eq 1 in which $R_{1 A}, \Delta \omega, f_{\mathrm{b}}$, and $\omega_{1}$ were provided to the fit. For each hydroxyl proton group in monosaccharides as assigned by NMR (i.e., $0.66,1.28,2.08$, and $2.88 \mathrm{ppm}$ ), an additional $\bar{R}_{\mathrm{ex}}$ term was added to $\overline{R_{1} \rho}$ in eq 1 . Glucose and $2-\mathrm{DG}$ have in total 4 different exchanging resonances; therefore, $4 \bar{R}_{\mathrm{ex}}$ terms were added to eq 1 . The mathematical equation, which relates $\overline{R_{1}}$ with $\bar{R}_{\mathrm{ex}}$, was described in the previous work and is provided in the SI Section 1.

Finally, the same $Z$-spectra (i.e., 77 frequency offsets ranging between $\pm 6.08 \mathrm{ppm}$ ) were fitted using a six-site exchange model for comparison (eqs 2 and 3). To calculate the intermembrane exchange rate, the sum $M_{\text {wzextralip }}^{\text {ss }}+M_{\text {wzintra }}^{\text {ss }}$ was fitted at 11 irradiation amplitudes. More precisely, $M_{\mathrm{wzextralip}}^{\mathrm{ss}}$ is substituted by eq 2 and the intraliposomal magnetization by eq 1 (see S17). For the fit, the equilibrium intraliposomal and extraliposomal water magnetizations were set as 15 and $85 \%$, respectively. $R_{1 A}, \Delta \omega, f_{\mathrm{b}}$, and $\omega_{1}$ were also provided to the fit as well as the frequency offsets and the relative proton concentrations of the hydroxyl groups in glucose or 2-DG. The error was calculated at the $95 \%$ confidence interval around the mean value.

The data are presented either in the form of normalized $Z$ spectra as a function of the saturation pulse frequency or as the magnetization transfer ratio asymmetry $\mathrm{MTR}_{\mathrm{asym}}$, which is defined as the difference in signal on either side of the water peak, which is centered at $0 \mathrm{ppm}$.

\section{RESULTS AND DISCUSSION}

Proton Assignment in Glucose and 2-DG. Figure 2 shows the high-resolution NMR spectra for glucose and 2-DG in the aqueous solution at $500 \mathrm{mM}$ and at various $\mathrm{pH}$ values. The peak assignments were performed by measurement of the downfield shifts compared to the water signal produced by the presence of $-\mathrm{OH}$ protons in glucose and 2-DG and by comparison to published values. ${ }^{27}$ The spectrum of glucose shows the resonances of the $-\mathrm{OH}$ protons, which correspond to signals at $0.66 \mathrm{ppm}\left(\mathrm{O}^{6} \mathrm{H}\right), 1.28 \mathrm{ppm}\left(\mathrm{O}^{2,3,4} \mathrm{H}\right)$ and the anomeric $-\mathrm{OH}$ protons at $2.08 \mathrm{ppm}\left(\mathrm{O}^{1} \mathrm{H}(\alpha)\right)$ and $2.88 \mathrm{ppm}$ $\left(\mathrm{O}^{1} \mathrm{H}(\beta)\right.$ ) (it is important to note that in the NMR data, positions are relative to tetramethylsilane and the resonance frequency of water is set at $4.75 \mathrm{ppm}$. However, in the $Z$ spectrum, the water frequency is referenced as $0 \mathrm{ppm}$, and the frequency offsets of the exchangeable protons groups are relative to $0 \mathrm{ppm}$ ). The spectrum of $2-\mathrm{DG}$ displays similar resonances as glucose but with the presence of only two $-\mathrm{OH}$ protons at $1.28 \mathrm{ppm}$. Figure 2 demonstrates that $\mathrm{pH}$ does not alter the resonant frequencies of the hydroxyl protons with respect to water. Similar NMR spectroscopy experiments were carried out with solutions of glucose and 2-DG at varying concentrations at $\mathrm{pH} 7$ resulting in similar findings (SI Figure $\mathrm{S} 1$ ). Thus, the hydroxyl peak assignment remains constant at different concentrations and $\mathrm{pH}$ and was in line with previously published results. $^{27}$ 
a
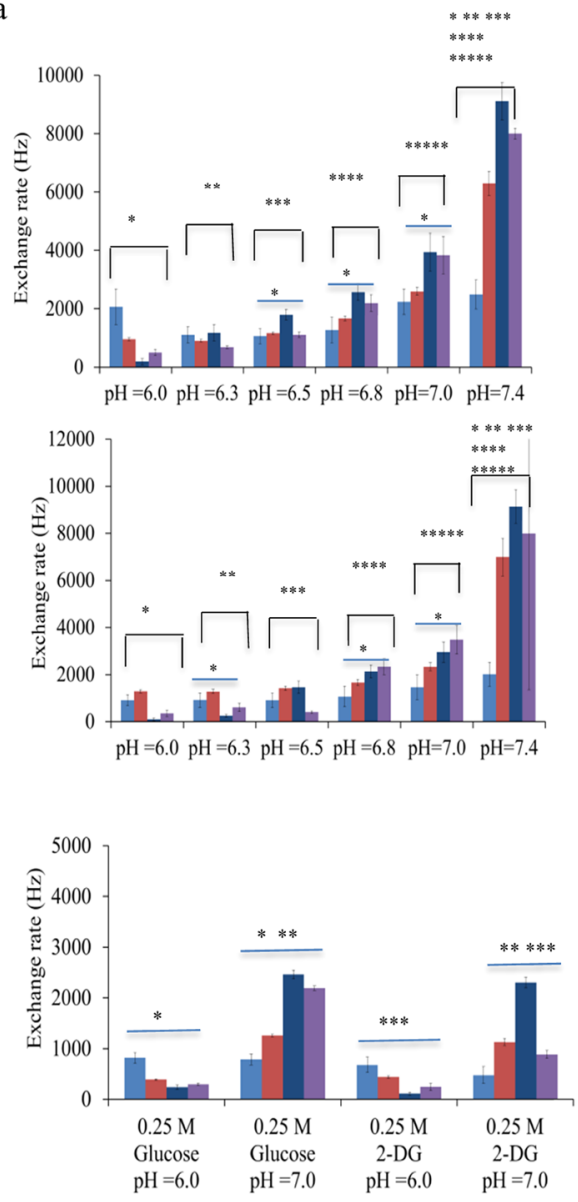

b

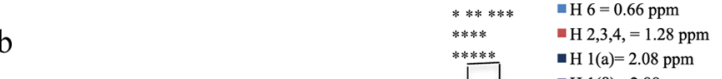

$\begin{array}{ll}* * * * * & -H 1(2)=2.08 \mathrm{ppm} \\ -\mathrm{H} l(\beta)=2.88 \mathrm{ppm}\end{array}$

d
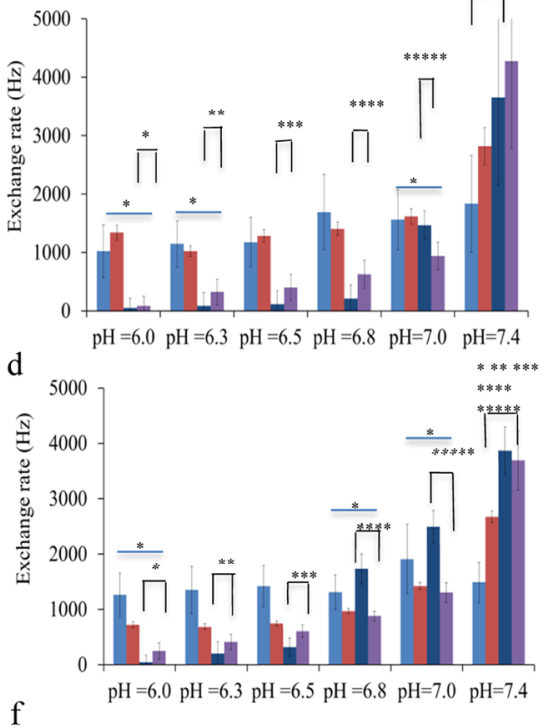

e

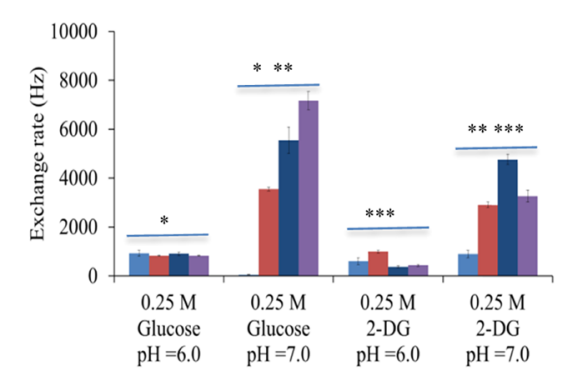

Figure 3. Experimentally measured exchange rates for $35 \mathrm{mM}$ free glucose scanned at $37{ }^{\circ} \mathrm{C}(\mathrm{a})$ and $25{ }^{\circ} \mathrm{C}(\mathrm{b})$ at various $\mathrm{pH}$ values in the range 6-7.4. The same procedure was performed for $35 \mathrm{mM}$ of 2-DG scanned at various $\mathrm{pH}$ values in the range $6-7.4$ and at $37^{\circ} \mathrm{C}(\mathrm{c})$ and $25^{\circ} \mathrm{C}(\mathrm{d})$. $0.25 \mathrm{M}$ of glucose and 2-DG at $\mathrm{pH}$ values 6 and 7 (e, f) are shown for comparison.

Exchange Rates of Free Monosaccharides at Various pH Values. Figure 3 displays the exchange rates of the hydroxyl protons in glucose (a, c) and 2-DG (b, d) at different $\mathrm{pH}$ values ranging between 6 and 7.4 at 25 and $37^{\circ} \mathrm{C}$. For quantification of the exchange rates, $Z$-spectra were collected at 11 irradiation amplitudes and fitted as detailed in the Experimental Section. Because of the large number of fitting variables, we fixed the known parameters such as the frequency offsets and the relative proton concentrations to improve the accuracy of the fitting algorithm.

The exchange of different hydroxyl groups in $35 \mathrm{mM}$ glucose and 2-DG belongs to the intermediate-fast exchange regime, and the experimentally measured exchange rates were found to be in the range of a few hundred $\mathrm{Hz}$ up to $10 \mathrm{kHz}$ depending on the $\mathrm{pH}$ and temperature of the sample (SI Tables S2-S5 and SI VII). At $\mathrm{pH} \mathrm{7,} \mathrm{the} \mathrm{exchange} \mathrm{of} \mathrm{glucose} \mathrm{and} \mathrm{2-DG}$ hydroxyl protons was occurring too rapidly so the detection of peaks in CEST spectra was no longer possible.

At physiologically relevant conditions (i.e., $\mathrm{pH} 7.4$ and 37 ${ }^{\circ} \mathrm{C}$ ), the exchange rates in glucose were measured as: $2.49 \mathrm{kHz}$ (0.66 ppm), $6.29 \mathrm{kHz}(1.28 \mathrm{ppm}), 9.11 \mathrm{kHz}(2.08 \mathrm{ppm})$, and $8.00 \mathrm{kHz}(2.88 \mathrm{ppm})$ (SI Table S3), whereas at $\mathrm{pH} 6$ and 37 ${ }^{\circ} \mathrm{C}$ were: $2.01 \mathrm{kHz}(0.66 \mathrm{ppm}), 0.95 \mathrm{kHz}(1.28 \mathrm{ppm}), 0.19$ $\mathrm{kHz}(2.08 \mathrm{ppm})$, and $0.50 \mathrm{~Hz}(2.88 \mathrm{ppm})$ (SI Table S3). For both monosaccharides, the exchange rate increased with an increase in temperature and $\mathrm{pH}$. At $25{ }^{\circ} \mathrm{C}$ and $\mathrm{pH} 6-6.5$, the protons at 0.66 and $1.28 \mathrm{ppm}$ exchange faster, whereas at $\mathrm{pH}$ 7.0 and 7.4, the exchange of the anomeric protons becomes the fastest. No significant differences were detected for the exchange rates of glucose and 2-DG at physiologically relevant conditions; however, at $\mathrm{pH} 6.25$ and 6.5, the exchange of anomeric protons was found to be significantly different between the two monosaccharides (SI Tables S3 and S5).

Data were also collected from $0.25 \mathrm{M}$ solutions of monosaccharides at 25 and $37{ }^{\circ} \mathrm{C}$ and at $\mathrm{pH} 6.0$ and 7.0 to assess whether there was any discrepancy between the measured exchange rates due to back-exchange of protons from water to monosaccharides, which might become important with the increase in the concentration (SI Tables S6 and S7). Note that $0.25 \mathrm{M}$ of glucose or 2-DG is approximately equal to the concentration of encapsulated monosaccharide inside the liposomes after dialysis (we use the calculated internal volume and measured overall monosaccharide concentration to estimate this internal concentration). As our results show (Figure $3 \mathrm{e}, \mathrm{f}$ ), the exchange rates are in the same order of magnitude but are significantly larger for $0.25 \mathrm{M}$ 2-DG and glucose at $\mathrm{pH} 7.0$ and $37^{\circ} \mathrm{C}$ when compared to 35 $\mathrm{mM}$ monosaccharide solutions under the same conditions. In addition, the same trend was observed for the measured exchange rates at $25{ }^{\circ} \mathrm{C}$. From these results, we can conclude that the effect of back-exchange of water protons becomes important at concentrations as high as $0.25 \mathrm{M}$ by over- 


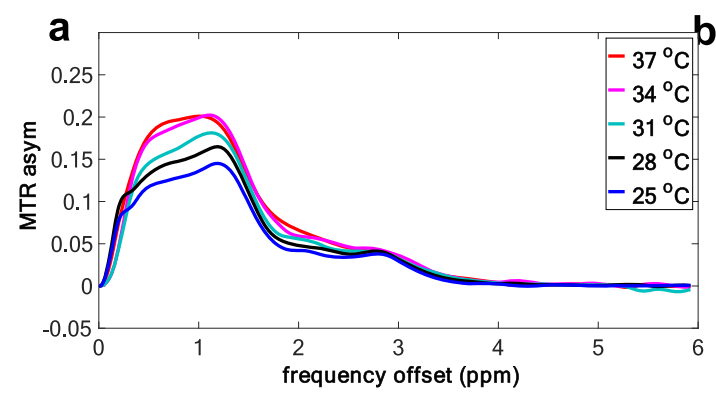

C

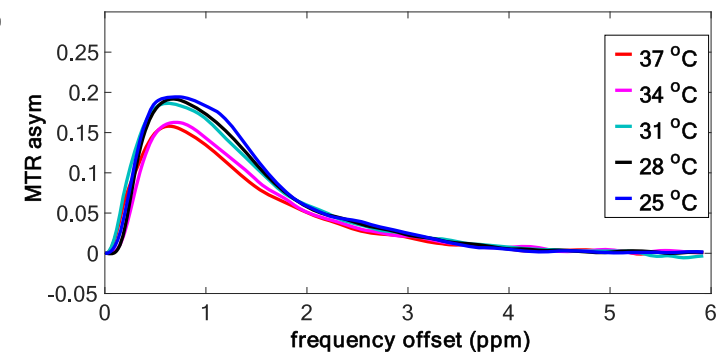

d
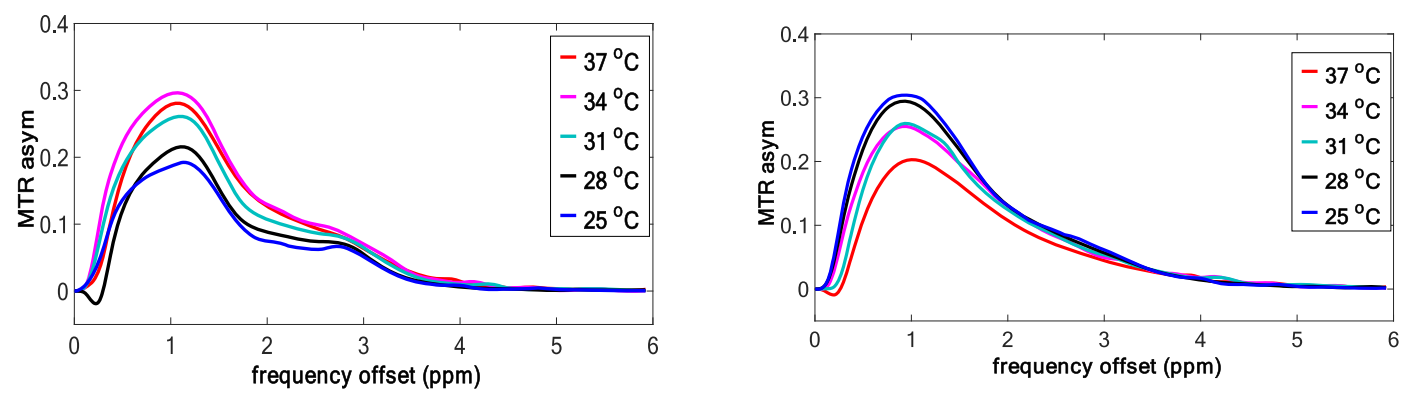

Figure 4. MTRasym spectra for gluco-liposome samples taken from formulation 1 (a, c) and free glucose at the same $\mathrm{pH}$ and concentration (d, b) scanned at $25,28,31,34$, and $37^{\circ} \mathrm{C}$ at power levels of $1.5 \mu \mathrm{T}(\mathrm{a}, \mathrm{b})$ and $5.0 \mu \mathrm{T}(\mathrm{c}, \mathrm{d})$.

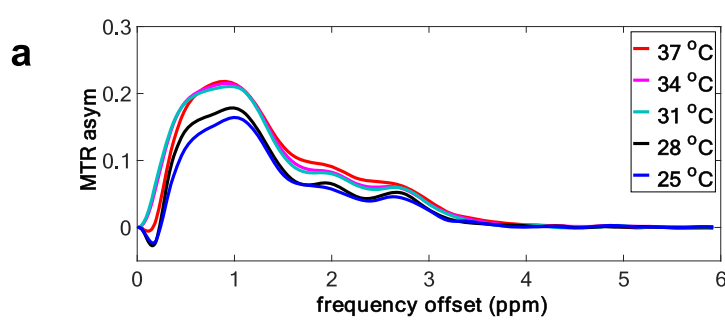

C

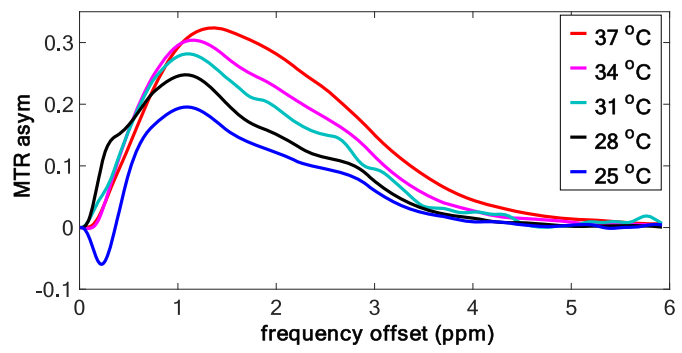

b

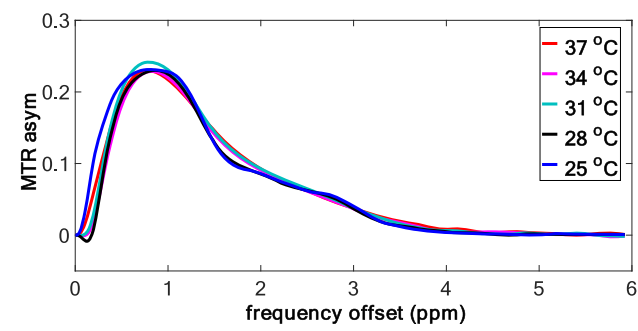

d

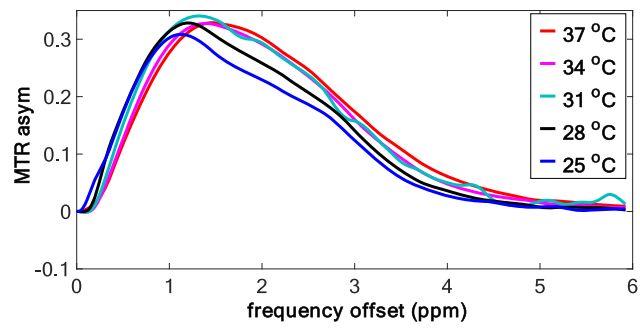

Figure 5. MTRasym spectra for 2-DG liposome samples from formulation 2 (a, c) and free 2-DG at the same $\mathrm{pH}$ and concentration (b, d) scanned at $25,28,31,34$, and $37^{\circ} \mathrm{C}$ at power levels of $1.5 \mu \mathrm{T}(\mathrm{a}, \mathrm{b})$ and $5.0 \mu \mathrm{T}(\mathrm{c}, \mathrm{d})$.

estimating the exchange rates of different hydroxyl groups depending on their relative concentration fractions.

Comparison of Chemical Exchange between Monosaccharides in Free Solutions and Encapsulated Monosaccharides Inside Liposomes. Figures 4 and 5 show the MTRasym spectra from liposome samples 1 and 2 encapsulating glucose and 2-DG, respectively, and the MTRasym obtained from free monosaccharides solutions with the same overall concentration and $\mathrm{pH}$. The liposome encapsulating monosaccharide spectra (Figures 4a,c and 5a,c) appear to display peaks, which are more pronounced at low powers of up to $1.57 \mu \mathrm{T}$, whereas the free monosaccharide spectra appear to have no visible peaks.

The presence of peaks in the liposomal samples is indicative of reduced chemical exchange rate compared to the free solution of monosaccharide with the same overall glucose concentration and scanned under the same conditions. This was further confirmed by comparing the measured exchange rates using a five-site exchange model. At $\mathrm{pH} 7.0$ and $37^{\circ} \mathrm{C}$, the exchange rates for the different hydroxyl groups in liposomes encapsulating 2-DG (formulation 2) were measured to be equal with: $1.20 \mathrm{kHz}(0.66 \mathrm{ppm}), 1.67 \mathrm{kHz}(1.28 \mathrm{ppm})$, $0.29 \mathrm{kHz}(2.08 \mathrm{ppm})$, and $1.39 \mathrm{kHz}(2.88 \mathrm{ppm}$ ) (SI Table S13). In addition, for gluco-liposomes (formulation 1), the exchange rates were measured as follows: $1.43 \mathrm{kHz}(0.66$ ppm), $1.57 \mathrm{kHz}$ (1.28 ppm), $0.75 \mathrm{kHz}$ (2.08 ppm), and 0.69 $\mathrm{kHz}$ (2.88 ppm) (SI Table S9). In summary, chemical exchange of glucose inside liposomes is quenched by one order of magnitude, if we looked at the experimentally calculated exchange rates at $25{ }^{\circ} \mathrm{C}$ from Tables S16 and S17, which is further confirmed by the measured exchange rates in 
a

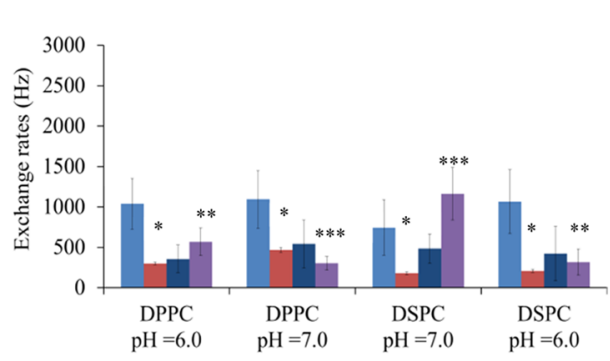

c
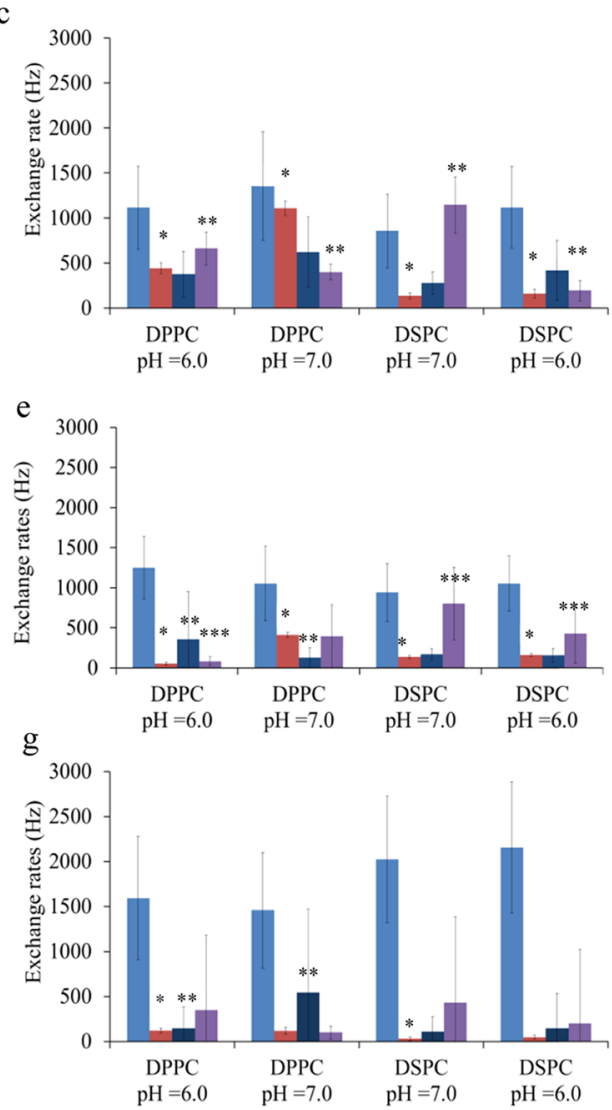
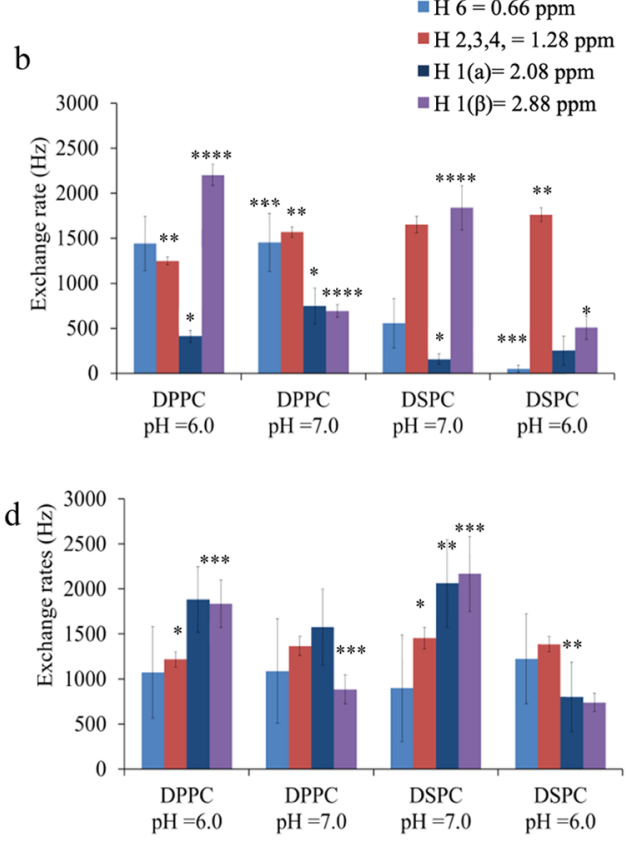

$\mathrm{f}$
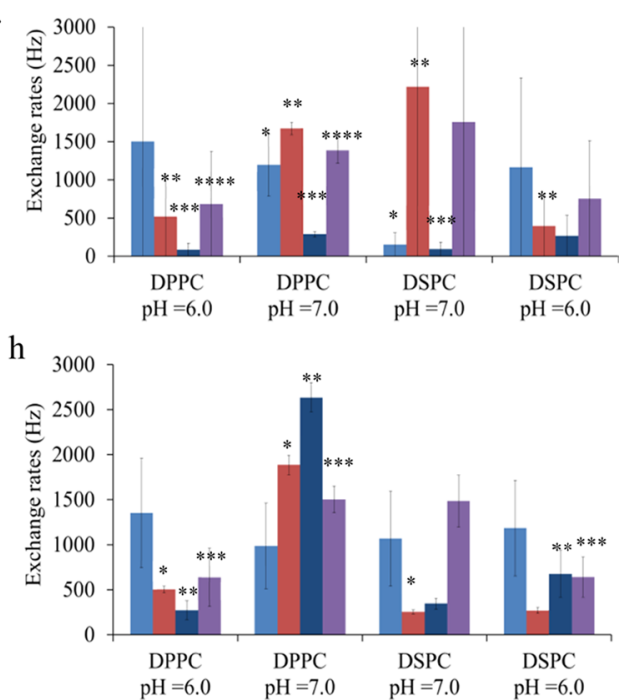

Figure 6. Experimentally measured exchange rates for DPPC vs DSPC liposomes encapsulating glucose (a-d, formulations 3, 4, 7, and 8) or 2-DG (e-h, formulations $5,6,9$, and 10 ) at $\mathrm{pH} 6$ or 7 and at $25^{\circ} \mathrm{C}(\mathrm{a}, \mathrm{c}, \mathrm{e}, \mathrm{g})$ or $37^{\circ} \mathrm{C}(\mathrm{b}, \mathrm{d}, \mathrm{f}, \mathrm{h})$ using either a five-site exchange model (a, b, e, f) or a six-site exchange model (c, d, g, h).

Tables S2 and S10. However, when the temperature is risen, this difference becomes half.

When calculating the exchange rates for liposome samples, we have assumed that a large majority of monosaccharide remains inside the liposomes throughout CEST MRI data acquisition. To confirm that this is the case, we conducted release over time studies at $37{ }^{\circ} \mathrm{C}$ (Supporting Information, Section VI) on $100 \%$ DPPC liposomes encapsulating $30 \mathrm{mM}$ 2-DG (formulation L3, Table S20). As liposomes incorporating PEG-lipids to coat the surface are known to be stabilized and to have a significantly longer in vivo half-life, ${ }^{28}$ we also

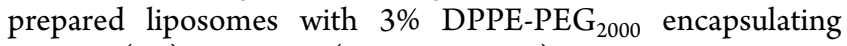
glucose (L1) or 2-DG (L2, Table S20). Our results showed that after incubation at $37{ }^{\circ} \mathrm{C}$ for $2 \mathrm{~h}$, the duration of the longer experiment, approximately $15 \%$ of the encapsulated 2 DG was released from the unshielded liposomes L3. By comparison, $1.4 \%$ of encapsulated glucose and $10 \%$ of the encapsulated 2-DG were released from the PEGylated liposomes L1 and L2, respectively (Supporting Information, Figure S2). For the range of overall concentrations exhibited by liposomal formulations $1-10$, this always equates to less than $5 \mathrm{mM}$ monosaccharide concentration exterior to the liposomes, which is approximately equal to the CEST detection limit for free glucose and 2-DG. Thus, we concluded that this quantity of leakage is negligible. In addition, the Glucose GO Assay Kit was used to check the overall and exterior glucose and 2-DG concentrations of liposome preparations, following the protocols described in the Supporting Information, after scanning. For samples that were scanned for less than $2 \mathrm{~h}$, no substantial leakage was detected. Consequently, we have assumed that a model of fivesite exchange or six-site exchange can be used to describe the liposomal system. 


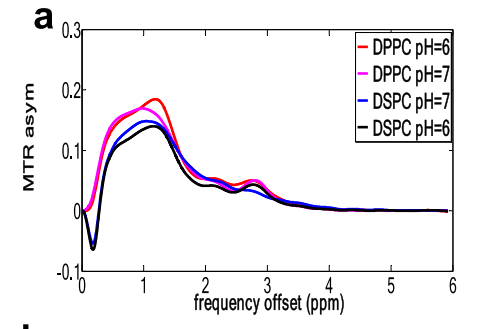

d

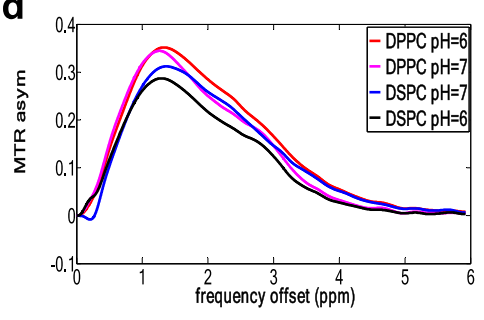

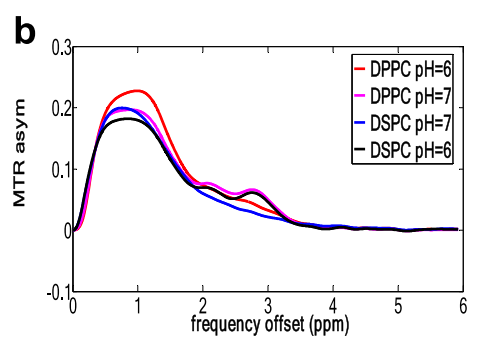

e

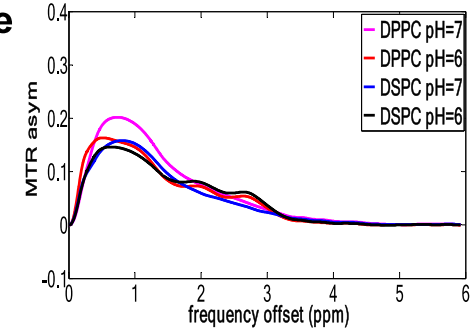

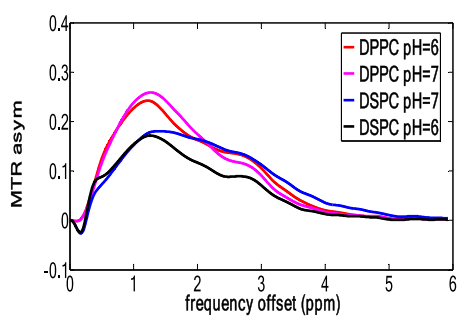

f

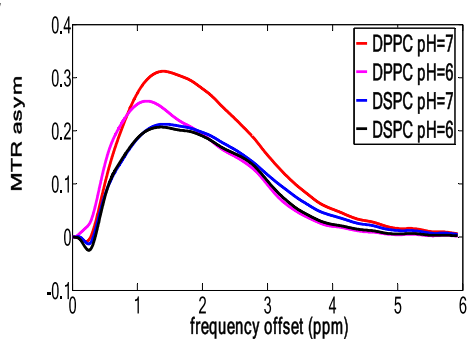

Figure 7. MTR asymmetry spectra of DPPC vs DSPC liposomes at 25 or $37{ }^{\circ} \mathrm{C}$. MTRasym spectra from DPPC and DSCP liposomes encapsulating glucose at $1.5 \mu \mathrm{T}(\mathrm{a}, \mathrm{c}, \mathrm{e})$ and $5.0 \mu \mathrm{T}(\mathrm{b}, \mathrm{d}, \mathrm{f})$ presaturation power at 25 and $37 \mathrm{C}$. The MTRasym from 2-DG DPPC, DSPC liposomes at $37 \mathrm{C}$ are also shown for comparison.

In principle, the CEST contrast arising from the glucoliposomal samples is due to the chemical exchange of glucose hydroxyl protons with the intraliposomal water and physical exchange between the intraliposomal and extraliposomal water, which falls under the slow exchange regime. ${ }^{19}$ As discussed earlier, the sample fraction made up by intraliposomal water is only $10-13 \%$ ( $87-90 \%$ is bulk extraliposomal water), thus the rate at which water enters/exits the intraliposomal space determines the ability of glucose to saturation-label bulk water protons. Consequently, $13 \%$ of the water $M_{0}$ is in direct exchange with glucose, whereas the remaining $87-90 \%$ interacts with glucose at a rate-limiting factor. Thus, the chemical exchange is severely reduced because of water compartmentalization compared to the free system of glucose in water. In the hypothetical case of a completely isolated system where the membrane would not allow water to diffuse across it, chemical exchange labeling would be confined to the small fraction of the sample water that is encapsulated inside the liposomes.

Effect of Physicochemical Properties of Liposomes on the Measured Exchange of the Encapsulated Contrast Agent. To further investigate the effect of the intermembrane exchange rate on the exchange of the encapsulated contrast agent, additional experiments were carried out with liposomes formed with two commercially available lipids, DPPC and DSPC. DSPC has two $\mathrm{C}_{18}$ saturated acyl chains compared to the two $\mathrm{C}_{16}$ saturated acyl chains of DPPC. This chain extension of only 2 carbons causes a drastic increase in the lipid phase transition temperature $\left(T_{\mathrm{m}}\right)$ from 41 ${ }^{\circ} \mathrm{C}$ for DPPC to $55^{\circ} \mathrm{C}$ for DSPC. ${ }^{17}$

From previous studies, it is evident that the membrane permeability and the phase transition profile are closely linked. Below the $T_{\mathrm{m}}$ of a lipid, the transfer of water across the bilayer is rate limiting and studies calculated that the water permeability at $26.5{ }^{\circ} \mathrm{C}$ is $0.5 \times 10^{-4} \mathrm{~cm} \mathrm{~s}^{-1}$, which increases to $26 \mathrm{~cm} \mathrm{~s}^{-1}$ at $45{ }^{\circ} \mathrm{C}$. ${ }^{29,30}$ We hypothesized that the more rigid bilayer formed by DSPC compared to DPPC will further reduce the transfer time of saturated protons from encapsulated monosaccharide to the bulk water due to alterations in physical water exchange across the bilayer. Figure 6 displays the measured exchange rates of DPPC and DSPC liposomes
2-10 encapsulating glucose or 2-DG. Exchange rates were measured for each liposomal system at $\mathrm{pH} 6.0$ (formulations 3, 5, 7, and 9 Table 1) and $\mathrm{pH} 7$ (formulations 4, 6, 8, and 10 Table 1) as well as at 25 and $37{ }^{\circ} \mathrm{C}$ (SI Tables S8-S15).

The MTR asymmetry spectra for DPPC and DSPC liposomes encapsulating monosaccharides are also displayed in Figure 7. According to our results, the chemical exchange rates of DPPC liposomes encapsulating monosaccharides at $\mathrm{pH} 6.0$ were found to be reduced compared to the results at $\mathrm{pH} 7.0$, as was expected. However, the calculated intermembrane exchange rates of DPPC and DSPC liposomes are within the errors. Thus, the effect of different lipid membranes can only be inspected by measuring the chemical exchange rates of monosaccharides encapsulated inside liposomes. According to the literature, at $37{ }^{\circ} \mathrm{C}$, the exchange rate of water across a DPPC bilayer is equal to $4.4 \mathrm{~Hz}$, whereas for the same conditions, it was found to be equal to $1.48 \mathrm{~Hz}$ for DSPC lipids. ${ }^{16}$ In addition, studies showed that the exchange rate of water across a DPPC bilayer reduces linearly with a reduction in temperature, whereas for DSPC lipids, it deviates from linearity. ${ }^{16}$ Thus, the exchange rates of monosaccharides encapsulated inside liposomes should be reduced when the scanning temperature is reduced from 37 to $25^{\circ} \mathrm{C}$. Finally, it is worth noting that when comparing the results of the DPPC liposomal samples encapsulating monosaccharides with those of free sugar solutions at $\mathrm{pH} 6.0$ and 7.0, liposome formulation is more "efficient" in quenching the signal for fast exchanging species (i.e., the exchange rates at $\mathrm{pH} 7.0$ were more reduced compared to the ones at $\mathrm{pH} 6.0$ ).

The temperature-induced changes to water exchange across the lipid bilayer were explored using DPPC liposomes 1 (encapsulating glucose) and 2 (encapsulating 2-DG), which were split up into 5 equivalent batches with exactly the same overall glucose or 2-DG concentrations. Each liposome batch and a free monosaccharide control were scanned at a single temperature to avoid cumulative release of liposomal contents, which would occur if a single sample was scanned at all ascending temperatures. Data were collected at 25, 28, 31, 34, and $37^{\circ} \mathrm{C}$ and are displayed in the form of MTRasym spectra (Figures 4 and 5). 
According to our results (Figures 4, 5, 8 and SI Tables S16S19), chemical exchange increases with an increase in

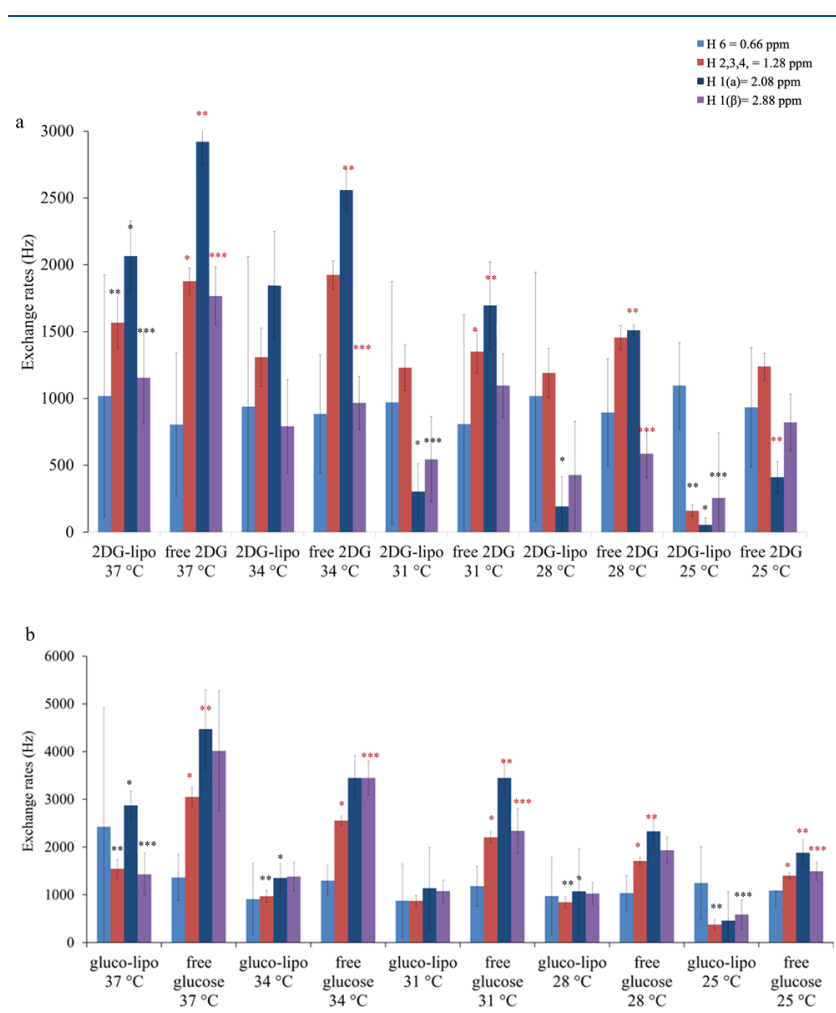

Figure 8. Exchange rates of gluco-liposomes at different temperatures. Experimentally measured exchange rates for gluco/2-DG liposomes and free 2-DG and glucose scanned at the same conditions for different temperatures ranging between 25 and $37^{\circ} \mathrm{C}$.

temperature both in the liposomal system and in free 2-DG and glucose. This is in line with previously published results in which changes in temperature resulted in an increase in the exchange rate of water across the bilayer by about $2-3$ orders of magnitude ${ }^{30}$ and to flatten spectra obtained from free 2-DG and glucose at $37{ }^{\circ} \mathrm{C}$. ${ }^{2,4}$ Below their $T_{\mathrm{m}}$, lipids exist in an ordered gel phase, but as they approach their $T_{\mathrm{m}}$, they assume an intermediate pretransition state (also termed the ripple phase), and finally at temperatures above the $T_{\mathrm{m}}$, they enter a disordered fluid state associated with trans-gauche isomerization. $^{30}$ Differential scanning calorimetry, electron spin resonance, and Laurdan fluorescence experiments have demonstrated that the pretransition phase can be characterized by up to $20 \%$ of the lipid population existing in the fluid state, and that DPPC bilayers enter this state at temperatures as low as $32{ }^{\circ} \mathrm{C} .{ }^{31,32}$ The measured differences in the exchange rates of the liposomal system at various temperatures can, thus, be partly attributed to these alterations in bilayer fluidity, i.e., higher temperatures result in an increased bilayer fluidity, which enhances physical exchange of water across the membrane and the transfer of saturated magnetization protons from monosaccharides to the extraliposomal bulk water. However, changes in temperature can also affect the longitudinal and transverse relaxation times of water, which further enhances the CEST contrast. In particular, a change from 25 to $37{ }^{\circ} \mathrm{C}$ produces an increase in $T_{1}$ from 3 to $4.2 \mathrm{~s}$ for a $9.4 \mathrm{~T}$ scanner resulting in more pronounced MTRasym spectra.
Calculation of Intermembrane Exchange Rate and Membrane Permeability for Different Lipid Compositions and Temperatures. Tables 2 and 3 present the measurements of the intermembrane exchange rate and the membrane permeabilities for different liposome compositions calculated as $P=\frac{R_{\text {interm }}}{3 r}$ where $r$ is the radius of the liposomes and $R_{\text {interm }}$ is the intermembrane exchange rate. According to

Table 2. Calculation of Intermembrane Exchange Rates and Membrane Permeability for DPPC and DSPC Liposomes under Different Conditions

\begin{tabular}{ccccc}
$\begin{array}{c}\text { lipid } \\
\text { bilayer }\end{array}$ & $\mathrm{pH}$ & $\begin{array}{c}\text { temperature } \\
\left({ }^{\circ} \mathrm{C}\right)\end{array}$ & $\begin{array}{c}\text { membrane } \\
\text { intermembrane } \\
\text { exchange rate }(\mathrm{Hz})\end{array}$ & $\begin{array}{c}\text { mermeability } \\
\left(\times 10^{-3} \mathrm{~cm} \mathrm{~s}^{-1}\right)\end{array}$ \\
DPPC & 7 & 25 & $50 \pm 64$ & 0.30 \\
DPPC & 6 & 25 & $52 \pm 59$ & 0.31 \\
DSPC & 7 & 25 & $39 \pm 45$ & 0.23 \\
DSPC & 6 & 25 & $44 \pm 44$ & 0.26 \\
DPPC & 7 & 37 & $95 \pm 134$ & 0.57 \\
DPPC & 6 & 37 & $61 \pm 63$ & 0.36 \\
DSPC & 7 & 37 & $66 \pm 79$ & 0.40 \\
DSPC & 6 & 37 & $59 \pm 67$ & 0.35 \\
\hline
\end{tabular}

Table 3. Calculation of Intermembrane Exchange Rates and Membrane Permeability for DPPC Liposomes at Various Temperatures

$\begin{array}{ccccc}\begin{array}{c}\text { lipid } \\ \text { bilayer }\end{array} & \mathrm{pH} & \begin{array}{c}\text { temperature } \\ \left({ }^{\circ} \mathrm{C}\right)\end{array} & \begin{array}{c}\text { membrane } \\ \text { intermembrane } \\ \text { exchange rate }(\mathrm{Hz})\end{array} & \begin{array}{c}\text { mermeability } \\ \text { perm } \\ \left(\times 10^{-3} \mathrm{~cm} \mathrm{~s}^{-1}\right)\end{array} \\ \text { DPPC } & 7 & 25 & 45 \pm 46 & 0.27 \\ \text { DPPC } & 7 & 28 & 56 \pm 78 & 0.34 \\ \text { DPPC } & 7 & 31 & 65 \pm 13 & 0.39 \\ \text { DPPC } & 7 & 34 & 79 \pm 66 & 0.47 \\ \text { DPPC } & 7 & 37 & 92 \pm 34 & 0.55\end{array}$

our results, the physical exchange of water across the bilayer for DPPC and DSPC lipids is in the slow exchange regime. This is in line with previous studies in which the intermembrane exchange rates were calculated in the presence of a paramagnetic contrast agent. ${ }^{31,32}$ It is important to note that small molecules such as glucose and 2-DG were encapsulated inside the liposomes; however, the general principles presented here could be applied to other diamagnetic substances, which possess exchangeable protons. In the same context, another technique for measuring the intermembrane exchange rate without the need of paramagnetic contrast agents is based on restricted diffusion of water molecules within cells termed filter exchange spectroscopy (FEXI). ${ }^{33}$ FEXI is promising for calculating exchange rates up to $40 \mathrm{~Hz}$; however, there is a lack of mathematical models that combine diffusion and the chemical exchange of CEST agents encapsulated inside liposomes. Here, we provide the mathematical equations, which describe a six-site exchange model in the case of a pulsed CEST experiment.

Five-Site vs Six-Site Exchange Model. A physical picture for the exchange rate dependence of the diaCEST liposomal contrast emerges from our analysis. For a five-site exchange model, we assumed that the intraliposomal water has the same magnetic properties as bulk water and a simplistic five-site exchange model was used to estimate the apparent exchange rate. When considering this type of analysis, the physical transfer of water across the bilayer has not been 
separated from the chemical exchange in the interior cavity. We assumed that the restrictions imposed by the lipid membrane will be indirectly measured by calculating the exchange rates of the encapsulated monosaccharide. On the contrary, a six-site exchange model separates the two processes. This can be done by taking the sum of the intraliposomal and extraliposomal water magnetizations weighted by their equilibrium values, which leads to a biexponential function (see eqs 2 and 3). More precisely, when fitting eqs 2 and 3 to the experimental data, the intermembrane exchange rate of water across the bilayer can be measured. According to our results, the exchange rates of monosaccharides calculated by a six-site exchange model are significantly larger than the ones calculated with a five-site exchange model (Figure 6). However, when using both models, the chemical exchange is happening slower for the liposomal system comparative to free monosaccharide solutions. It should be noted that we excluded any effects of water diffusion from our measurements, and any alterations in the CEST signal of the liposomal samples were solely attributed to a difference in the chemical exchange rate. This was obvious from simulated data generated at different $T_{2}$ values of the intraliposomal and extraliposomal water magnetization. In particular, the simulated data matched the experimental results only when the $T_{2}$ values of the two compartments were identical. In addition, experimentally calculated $T_{2}$ values of the liposomal samples encapsulating monosaccharides were found to be equal to the $T_{2}$ values of free monosaccharides. In turn, a negligible change in $T_{2}$ implies no diffusivity changes for the liposomal system.

Our theoretical work was further validated by numerically solving the BM equations for both models (see SI, Figures S3S6). According to our results, the liposomal system produces a more pronounced CEST effect compared to the solution of free monosaccharides, and CEST contrast does not change for intermembrane exchange rates exceeding $100 \mathrm{~Hz}$. Another finding was that the chemical exchange of monosaccharide with intraliposomal water produces a much broader CEST effect in the interior cavity compared to the measured CEST effect in the extraliposomal space. In particular, CEST contrast enhancement inside the liposomes is produced by using as high as $0.25 \mathrm{M}$ concentration of monosaccharide, which, in turn, reduces the intraliposomal water content to $91 \%$. At the same time, back-exchange of water protons to monosaccharide hydroxyl proton could explain why different hydroxyl groups on the encapsulated sugars are affected in different ways. Further inspection of our fitting results shows that the calculated exchange rate was found to be equal with $50 \mathrm{~Hz}$ (0.66 ppm, Table S7), which might be indeed a local minimum since the fitting reached the boundary conditions. However, the quality of the fitting is very good (zero residual between the fitted and experimental data, SI VIII), which might indicate that calculation of $k_{\mathrm{BA}}$ does not produce significant errors for calculating the exchange rates at $1.28,2.08,2.88 \mathrm{ppm}$. To confirm that this is the case, we ran the fitting procedure again with different boundary conditions for the exchange rate at $0.66 \mathrm{ppm}$, and the calculated exchange rates are as follows: $k_{\mathrm{BA}}$ $=1384 \pm 438 \mathrm{~Hz}, k_{\mathrm{DA}}=2842 \pm 296 \mathrm{~Hz}, k_{\mathrm{EA}}=5583 \pm 564 \mathrm{~Hz}$, $k_{\mathrm{FA}}=8742 \pm 296 \mathrm{~Hz}$.

It is important to note that for our fitting, we used a least square approach and we fixed the known or measured experimental parameters such as the concentration, peak assignment, $\mathrm{T}_{2}$ of glucose, and fitted only the exchange rates within a range of $50-10000 \mathrm{~Hz}$. As we noticed the fitting approach is sensitive to the number of fitting parameters as well as boundary conditions. However, the calculated exchange rates are in line with the work conducted independently by other researchers using similar fitting approaches. ${ }^{34}$

Limitations. Here, we would like to discuss a few limitations of our approach. It is evident that the calculated intermembrane exchange rates are within the errors. Thus, no conclusions can be drawn when we assess the physical exchange of water across a DPPC or DSPC bilayer, for example. However, the difference in water transfer across the membrane consisting of DPPC or DSPC can be inspected by calculating the exchange rates of the encapsulated monosaccharides. This can be attributed to two reasons: (1) for intermembrane exchange rates of $100 \mathrm{~Hz}$ and above, the obtained $Z$-spectra overlap (refer to our simulations in the SI). Thus, for the bilayers we used here, we reached the limits of our technique. (2) The frequency offset of the water inside and outside the liposomes is the same and can only produce a slight effect on direct water saturation compared to free water (i.e., the case we scan an empty liposome). The initial equations derived for a two-site model ${ }^{21}$ (extended here to a five-site model) do not function properly close to water, thus it might not be possible to decouple two exchange processes at 0 ppm when the exchange of the solute (e.g., glucose or 2-DG) is happening too fast and the water exchange across the membrane at an intermediate exchange rate.

\section{CONCLUSIONS}

The potential benefits of liposomally delivered GlucoCEST and 2DG-CEST reagents include monitoring the uptake and accumulation of liposomes in tumors ${ }^{35}$ and probing alterations in brain 2DG uptake in neurodegenerative diseases. ${ }^{36}$ However, several challenges remain for clinical translation of GlucoCEST MRI including low signal, off-target effects, signal contamination by patient's motion, and violation of the slow to intermediate exchange condition for fast exchanging species such as glucose or 2-DG, especially for low field MRI scanners. Biocompatible agents such as liposomes have been proposed as a useful tool for encapsulating and delivering potentially toxic diaCEST agents to their targets, particularly when the purpose is to target and kill cancer cells. We hypothesized that developing a new generation of GlucoCEST contrast agents, encapsulating high concentrations of monosaccharides inside liposomes, could overcome and facilitate translation of GlucoCEST into the clinic.

The measurements presented here of the exchange rates of encapsulated monosaccharides inside liposomes improve not only our current understanding of the origin of the measured signal but also provide guidelines for better optimization of the CEST contrast. Our goal is to present a mechanistic picture of the effect of the diaCEST liposomal agents. Thus, we report the measured exchange rates of the gluco-liposomal system on a molecular basis by keeping the same intraliposomal water fraction and amount of encapsulated sugars in all our samples. In this study, we used a rich multipower dataset for calculating the exchange rates of monosaccharide hydroxyl pools inside liposomes. To be able to separate the effects of the chemical exchange from physical water transfer across the bilayer barrier, we constructed a six-site exchange model, which describes such systems in a much more precise way, as previously reported. Thus, improved estimates of the exchange rates of hydroxyl protons in liposomes are provided. This also allowed us to demonstrate that the effect of the slow physical transfer of 
water across the lipid membrane for different lipid composition and temperatures changes the exchange rates of the encapsulated monosaccharides inside liposomes. To the best of our knowledge, this is the first study in which a model has been constructed to measure the exchange properties of diaCEST agents encapsulated inside liposomes. ${ }^{37}$ Our theoretical development for quantifying the exchange rates of monosaccharides encapsulated in liposomes will be beneficial for future GlucoCEST studies, whereas the same principles can be applied for other diaCEST molecules. Moreover, the potential benefits of this approach include a more detailed understanding of the effects of membrane lipid composition on permeability in both model liposomes used to transport drugs or contrast agents and in membrane systems designed to mimic the composition of biological cell membranes. This will, in turn, have implications for the design of new liposomal drug carriers as well as a better understanding of drug toxicity, pharmacokinetics, and the factors governing membrane fluidity.

Importantly, we have also demonstrated that liposome encapsulation with DPPC of both glucose and 2-DG gives higher CEST signals compared with the free sugar solutions. This increase in signal for the liposomal system occurs due to a reduction in the exchange rate, which is more pronounced for 2DG liposomes compared to glucose liposomes. We also observe a 10-fold reduction in the exchange rate when we used DSPC, a rigid lipid, which quenches the intermembrane exchange across the lipid bilayer. Currently, glucoCEST is challenging at clinical field strengths of $3 \mathrm{~T}$ and lower: the chemical exchange of both glucose and 2-DG falls in the intermediate to fast exchange regime (as shown in this paper) resulting in a substantial signal decrease when translating this technique from mouse models to humans. These findings are, therefore, significant, as liposomal encapsulation has the potential to make GlucoCEST much easier and reproducible to carry out at clinical field strengths. The enhancement of CEST signal was observed at both $\mathrm{pH} 6$ and $\mathrm{pH}$ 7; however, the chemical exchange of the encapsulated monosaccharides is also affected by modulating the $\mathrm{pH}$ of the contrast agent, suggesting that this technique could further be developed to report on the tumor microenvironment. In future work, we aim to validate the developed contrast agents in various in vivo cancer models and compare these experiments with standard GlucoCEST techniques.

\section{ASSOCIATED CONTENT}

\section{S Supporting Information}

The Supporting Information is available free of charge on the ACS Publications website at DOI: 10.1021/acs.jpcb.9b02280.

Calculations: derivation of a six-site exchange model; calculation of encapsulation efficiency; determination of monosaccharide concentrations of liposomal samples; NMR shifts of hydroxyl protons in glucose and 2-DG at varying concentrations; results of exchange rates for free monosaccharides and monosaccharides encapsulated inside liposomes with five and six-site exchange model; five-site vs six-site exchange model; six-site model; fivesite model; six-site model; release over time experiments; simulations of the liposomal system (PDF)

\section{AUTHOR INFORMATION}

\section{Corresponding Authors}

*E-mail: eleni.demetriou.13@ucl.ac.uk (E.D.).

*E-mail: a.b.tabor@ucl.ac.uk (A.B.T.).

\section{ORCID}

Helen C. Hailes: 0000-0001-5574-4742

Alethea B. Tabor: 0000-0001-8216-0347

\section{Author Contributions}

${ }^{\S}$ E.D. and H.E.S. contributed equally and wish to be considered joint first authors.

\section{Notes}

The authors declare no competing financial interest.

\section{ACKNOWLEDGMENTS}

We gratefully acknowledge the European Union's Horizon 2020 research and innovation programme (Grant Agreement No. 667510 to X.G.) and UCL Grand Challenges scheme. This work was conducted within the King's College London-UCL Comprehensive Cancer Imaging Centre (CCIC) supported by Cancer Research U.K. and the EPSRC, in association with MRC and DoH (U.K.), and supported by researchers at the National Institute for Health Research University College London Hospitals Biomedical Research Centre. The authors would like to thank Cancer Research U.K. and the EPSRC (grant C5255/A15935 (R.B.) and studentship EP/M506448/1 (H.S.)) for financial support. We would like to thank Professor Jayne Lawrence, Dr. Hisham Al-Obaidi and Dr. Fabrizia Foglia (Institute of Pharmaceutical Science, King's College London) for access to their LIPEX extruder and Zetasizer, and also to Professor Giuseppe Battaglia (Department of Chemistry, UCL) for access to the Zetasizer, and to both groups for many helpful discussions.

\section{REFERENCES}

(1) van Zijl, P. C. M.; Yadav, N. N. Chemical Exchange Saturation Transfer (CEST): What is in a Name and What Isn't? Magn. Reson. Med. 2011, 65, 927-948.

(2) Walker-Samuel, S.; Ramasawmy, R.; Torrealdea, F.; Rega, M.; Rajkumar, V.; Johnson, S. P.; Richardson, S.; Gonçalves, M.; Parkes, H. G.; Årstad, E.; et al. In vivo imaging of glucose uptake and metabolism in tumors. Nat. Med. 2013, 19, 1067-1072.

(3) Nasrallah, F. A.; Pagès, G.; Kuchel, P. W.; Golay, X.; Chuang, K.$\mathrm{H}$. Imaging brain deoxyglucose uptake and metabolism by glucoCEST MRI. J. Cereb. Blood Flow Metab. 2013, 33, 1270-1278.

(4) Chan, K. W. Y.; McMahon, M. T.; Kato, Y.; Liu, G.; Bulte, J. W. M.; Bhujwalla, Z. M.; Artemov, D.; van Zijl, P. C. M. Natural Dglucose as a biodegradable MRI contrast agent for detecting cancer. Magn. Reson. Med. 2012, 68, 1764-1773.

(5) Rivlin, M.; Horev, J.; Tsarfaty, I.; Navon, G. Molecular imaging of tumors and metastases using chemical exchange saturation transfer (CEST) MRI. Sci. Rep. 2013, 3, No. 3045.

(6) Jin, T.; Mehrens, H.; Hendrich, K. S.; Kim, S. G. Mapping brain glucose uptake with chemical exchange-sensitive spin-lock magnetic resonance imaging. J. Cereb. Blood Flow Metab. 2014, 34, 1402-1410.

(7) Dwarakanath, B. S.; Singh, D.; Banergi, A. K.; Sarin, R.; Venkataramana, N. K.; Jalali, R.; Vishwanath, P. N.; Mohanti, B. K.; Tripathi, R. P.; Kalia, V. K.; et al. Clinical studies for improving radiotherapy with 2-deoxy-D-glucose: Present status and future prospects. J. Cancer. Res. Ther. 2009, 5, 21-26.

(8) Aime, S.; Delli Castelli, D.; Terreno, E. Highly sensitive MRI chemical exchange saturation transfer agents using liposomes. Angew. Chem., Int. Ed. 2005, 44, 5513-5515.

(9) Ferrauto, G.; Delli Castelli, D.; Di Gregorio, E.; Terreno, E.; Aime, S. LipoCEST and cellCEST imaging agents: opportunities and 
challenges. Wiley Interdiscip. Rev.: Nanomed. Nanobiotechnol. 2016, 8, 602-618.

(10) Chan, K. W. Y.; Bulte, J. W. M.; McMahon, M. T. Diamagnetic chemical exchange saturation transfer (diaCEST) liposomes: physicochemical properties and imaging applications. Wiley Interdiscip. Rev.: Nanomed. Nanobiotechnol. 2014, 6, 111-124.

(11) Blanco, E.; Shen, H.; Ferrari, M. Principles of nanoparticle design for overcoming biological barriers to drug delivery. Nat. Biotechnol. 2015, 33, 941-951.

(12) Hwang, J. Y.; Li, Z. B.; Loh, X. J. Small molecule therapeuticloaded liposomes as therapeutic carriers: from development to clinical applications. RSC Adv. 2016, 6, 70592-70615.

(13) Malam, Y.; Loizidou, M.; Seifalian, A. M. Liposomes and nanoparticles: nanosized vehicles for drug delivery in cancer. Trends Pharmacol. Sci. 2009, 30, 592-599.

(14) van Zijl, P. C. M.; Jones, C. K.; Ren, J.; Malloy, C. R.; Sherry, A. D. MRI detection of glycogen in vivo by using chemical exchange saturation transfer imaging (glycoCEST). Proc. Natl. Acad. Sci. U.S.A. 2007, 104, 4359-4364.

(15) Awoonor-Williams, E.; Rowley, C. N. Molecular simulation of nonfacilitated membrane permeation. Biochim. Biophys. Acta, Bioenerg. 2016, 1858, 1672-1687.

(16) Siontorou, C. G.; Nikoleli, G.-P.; Nikolelis, D. P.; Karapetis, S. K. Artificial Lipid Membranes: Past, Present, and Future. Membranes 2017, 7, 38.

(17) Mathai, J. C.; Tristram-Nagle, S.; Nagle, J. F.; Zeidel, M. L. Structural determinants of water permeability through the lipid membrane. J. Gen. Physiol. 2008, 131, 69-76.

(18) Terreno, E.; Sanino, A.; Carrera, C.; Delli Castelli, D.; Giovenzana, G. B.; Lombardi, A.; Mazzon, R.; Milone, L.; Visigalli, M.; Aime, S. Determination of water permeability of paramagnetic liposomes of interest in MRI field. J. Inorg. Biochem. 2008, 102, 11121119.

(19) Zhao, J. M.; Har-el, Y.-E.; McMahon, M. T.; Zhou, J.; Sherry, A. D.; Sgouros, G.; Bulte, J. W. M.; van Zijl, P. C. M. Size-induced enhancement of chemical exchange saturation transfer (CEST) contrast in liposomes. J. Am. Chem. Soc. 2008, 130, 5178-5184.

(20) Abergel, D.; Palmer, A. G. Approximate solutions of the BlochMcConnell equations for two-site chemical exchange. ChemPhysChem 2004, 5, 787-793.

(21) Meissner, J.-E.; Goerke, S.; Rerich, E.; Klika, K. D.; Radbruch, A.; Ladd, M. E.; Bachert, P.; Zaiss, M. Quantitative pulsed CEST-MRI using Omega-plots. NMR Biomed. 2015, 28, 1196-1208.

(22) Xu, X.; Khan, M. A.; Burgess, D. J. Predicting hydrophilic drug encapsulation inside unilamellar liposomes. Int. J. Pharm. 2012, 423, 410-418.

(23) Marra, J.; Israelachvili, J. Direct measurement of forces between phosphatidylcholine and phosphatidylethanolamine bilayers in aqueous-electrolyte solutions. Biochemistry 1985, 24, 4608-4618.

(24) Sun, W. J.; Tristram-Nagle, S.; Suter, R. M.; Nagle, J. F. Structure of gel phase saturated lecithin bilayers: Temperature and chain length dependence. Biophys. J. 1996, 71, 885-891.

(25) Tristram-Nagle, S.; Zhang, R.; Suter, R. M.; Worthington, C. R.; Sun, W. J.; Nagle, J. F. Measurement of chain tilt angle in fully hydrated bilayers of gel phase lecithins. Biophys. J. 1993, 64, 10971109.

(26) McComb, R. B.; Yuskok, W. D.; Batt, W. G. 2-Deoxy-Dglucose, a new substrate for glucose oxidase (glucose aerodehydrogenase). J. Franklin Inst. 1957, 263, 161-165.

(27) Bociek, S.; Franks, F. Proton-exchange in aqueous-solutions of glucose - hydration of carbohydrates. J. Chem. Soc., Faraday Trans. 1 1979, 75, 262-270.

(28) Klibanov, A. L.; Maruyama, K.; Torchilin, V. P.; Huang, L. Amphipathic polyethyleneglycols effectively prolong the circulation time of liposomes. FEBS Lett. 1990, 268, 235-237.

(29) Reeves, J. P.; Dowben, R. M. Water Permeability of Phospholipid Vesicles. J. Membr. Biol. 1970, 3, 123-141.
(30) Saito, H.; Shinoda, W. Cholesterol effect on water permeability through DPPC and PSM lipid bilayers: A molecular dynamics study. J. Phys. Chem. B 2011, 115, 15241-15250.

(31) Riske, K. A.; Barroso, R. P.; Vequi-Suplicy, C. C.; Germano, R.; Henriques, V. B.; Lamy, M. T. Lipid bilayer pre-transition as the beginning of the melting process. Biochim. Biophys. Acta, Biomembr. 2009, 1788, 954-963.

(32) Mason, P. C.; Gaulin, B. D.; Epand, R. M.; Wignall, G. D.; Lin, J. S. Small angle neutron scattering and calorimetric studies of large unilamellar vesicles of the phospholipid dipalmitoylphosphatidylcholine. Phys. Rev. E 1999, 59, 3361-3367.

(33) Åslund, I.; Nowacka, A.; Nilsson, M.; Topgaard, D. Filterexchange PGSE NMR determination of cell membrane permeability. J. Magn. Reson. 2009, 200, 291-295.

(34) Zaiss, M.; Anemone, A.; Goerke, S.; Longo, D. L.; Herz, K.; Pohmann, R.; Aime, S.; Rivlin, M.; Navon, G.; Golay, X.; Scheffler, K. Quantification of hydroxyl exchange of D-Glucose at physiological conditions for optimization of glucoCEST MRI at $3 \mathrm{~T}, 7 \mathrm{~T}$ and $9.4 \mathrm{~T}$. NMR Biomed. 2019, No. e4113.

(35) Chan, K. W. Y.; Yu, T.; Qiao, Y.; Liu, Q.; Yang, M.; Patel, H.; Liu, G. S.; Kinzler, K. W.; Vogelstein, B.; Bulte, J. W. M.; et al. A diaCEST MRI approach for monitoring liposomal accumulation in tumors. J. Controlled Release 2014, 180, 51-59.

(36) Tolomeo, D.; Micotti, E.; Serra, S. C.; Chappell, M.; Snellman, A.; Forloni, G. Chemical exchange saturation transfer MRI shows low cerebral 2-deoxy-D-glucose uptake in a model of Alzheimer's Disease. Sci. Rep. 2018, 8, No. 9576.

(37) Castelli, D. D.; Farrauto, G.; Terreno, E.; Aime, S. Chemical Exchange Saturation Transfer Imaging: Advances and Applications; McMahon, M. T., Gilad, A. A., Bulte, J. W. M., van Zijl, P. C. M., Eds.; Pan Stanford, 2017; pp 311-336. 